\title{
On the Hybrid Power Mean of Two-Term Exponential Sums and Cubic Gauss Sums
}

\author{
Shaofan Cao $i$ and Tingting Wang $(i)$ \\ College of Science, Northwest A\&F University, Yangling, Shaanxi, China \\ Correspondence should be addressed to Tingting Wang; ttwang@nwsuaf.edu.cn
}

Received 14 December 2020; Revised 31 March 2021; Accepted 10 May 2021; Published 30 May 2021

Academic Editor: Stanislaw Migorski

Copyright (C) 2021 Shaofan Cao and Tingting Wang. This is an open access article distributed under the Creative Commons Attribution License, which permits unrestricted use, distribution, and reproduction in any medium, provided the original work is properly cited.

In this paper, an interesting third-order linear recurrence formula is presented by using elementary and analytic methods. This formula is concerned with the calculating problem of the hybrid power mean of a certain two-term exponential sums and the cubic Gauss sums. As an application of this result, some exact computational formulas for one kind hybrid power mean of trigonometric sums are obtained.

\section{Introduction}

As usual, let $p$ be an odd prime. For any integer $m$, we define the cubic Gauss sums $A(m, p)=A(m)$ as follows:

$$
A(m)=\sum_{a=0}^{p-1} e\left(\frac{m a^{3}}{p}\right)
$$

where $e(y)=e^{2 \pi i y}$ and $i^{2}=-1$.

In recent years, several scholars have studied the hybrid power mean problems of various trigonometric sums and proved many interesting results. For example, Chen and $\mathrm{Hu}$ [1] studied the computational problem of

$$
S_{k}(p)=\sum_{m=1}^{p-1}\left(\sum_{a=0}^{p-1} e\left(\frac{\mathrm{ma}^{3}}{p}\right)\right)^{k} \cdot\left|\sum_{c=1}^{p-1} e\left(\frac{m c+\bar{c}}{p}\right)\right|^{2},
$$

where $\bar{c}$ denotes the multiplicative inverse of $c \bmod p$. That is, $c \cdot \bar{c} \equiv 1 \bmod p$. For $p \equiv 1 \bmod 3$, they obtained a thirdorder linear recurrence formula for $S_{k}(p)$.

$\mathrm{Li}$ and $\mathrm{Hu}[2]$ studied another hybrid power mean

$$
\sum_{b=1}^{p-1}\left|\sum_{a=0}^{p-1} e\left(\frac{\mathrm{ba}^{4}}{p}\right)\right|^{2} \cdot\left|\sum_{c=1}^{p-1} e\left(\frac{\mathrm{bc}+\bar{c}}{p}\right)\right|^{2}
$$

and gave an exact computational formula for (3).

Some other related papers can also be found in references [3-11]. We would not have to repeat them here.

Very recently, Chen and Chen [12] studied the recursive properties of the hybrid power mean

$$
H_{k}(c, p)=\sum_{m=1}^{p-1}\left(\sum_{a=0}^{p-1} e\left(\frac{\mathrm{cma}^{3}}{p}\right)\right)^{k} \cdot\left(\sum_{b=0}^{p-1} e\left(\frac{\mathrm{mb}^{3}+b}{p}\right)\right)^{3},
$$

and obtained a third-order linear recurrence formula for it with $c=1$ and $p \equiv 1 \bmod 3$. That is, they proved the following result:

Let $p$ be an odd prime with $p \equiv 1 \bmod 3$. If 3 is a cubic residue $\bmod p$, then for any integer $k \geq 3$, one has the thirdorder linear recurrence formula:

$$
H_{k}(1, p)=3 \mathrm{pH}_{k-2}(1, p)+\mathrm{dpH}_{k-3}(1, p),
$$


where the first three terms are $H_{0}(1, p)=2 p^{2}-\mathrm{pd}$, $H_{1}(1, p)=p^{2}(d-6)$, and $H_{2}(1, p)=p^{2}(6 p-5 d)$.

Note that Zhang and Zhang [13] proved the identity

$$
\sum_{m=1}^{p-1}\left|\sum_{a=0}^{p-1} e\left(\frac{\mathrm{ma}^{3}+\mathrm{na}}{p}\right)\right|^{4}= \begin{cases}2 p^{3}-p^{2} & \text { if } 3+p-1 \\ 2 p^{3}-7 p^{2} & \text { if } 3 \mid p-1\end{cases}
$$

Perhaps this is the best result for which there is no conditional requirement on the prime $p$. The interesting results of the above work motivate us to ask such a problem of whether there exists a similar recursive formula for the hybrid power mean

$$
U_{k}(p)=\sum_{m=1}^{p-1}\left(\sum_{a=0}^{p-1} e\left(\frac{\mathrm{cma}^{3}}{p}\right)\right)^{k} \cdot\left|\sum_{b=0}^{p-1} e\left(\frac{\mathrm{mb}^{3}+b}{p}\right)\right|^{4}
$$

where $p$ is an odd prime with $p \equiv 1 \bmod 3$.

Obviously, the problem in (7) is much harder than the problem in [12] because we are dealing with the fourth power mean of the two-term exponential sums in (7). Our main contribution is to obtain an identity for the fourth power mean of the two-term exponential sums weighted by third-order character modulo $p$, i.e., the following Lemma 3. Then, we use this lemma to derive several interesting recursion formulas for $U_{k}(p)$. In this way, the continuities and the value distribution properties of this kind of trigonometric sums can be described from different views. Of course, the reason why we focus on the calculation of (7) is that the problem is closely related to the number of the solutions of some congruence equation. These contents play a very important role in study of some famous analytic number theory problems, such as Waring problem and Goldbach conjecture.

Through the study, it is found that the problem we studied is closely related to integer 3 . If 3 is a cubic residue modulo $p$, then there exists a beautiful third-order linear recurrence formula for $U_{k}(p)$, and the first three terms $U_{0}(p), U_{1}(p)$, and $U_{2}(p)$ are integers. If 3 is not a cubic residue mod $p$, then we can get the exact value of $U_{2}(p)$. For any other positive integer $k$, we can only give a more complex mathematical representation for $U_{k}(p)$. That is, we have the following three results:
Theorem 1. Let $p$ be a prime with $p \equiv 1 \bmod 3$. If 3 is a cubic residue modulo $p$, then for any integer $k \geq 3$, we have the third-order linear recurrence formula

$$
U_{k}(p)=3 p U_{k-2}(p)+\mathrm{dpU}_{k-3}(p)
$$

where the first three terms are $U_{0}(p)=2 p^{3}-7 p^{2}$, $U_{1}(p)=-d p(6 p-1)$, and $U_{2}(p)=p^{2}\left(4 p^{2}-22 p-d^{2}+2\right)$ and $d$ is uniquely determined by $4 p=d^{2}+27 b^{2}$ and $d \equiv 1 \bmod 3$.

Theorem 2. Let $p$ be a prime with $p \equiv 1 \bmod 3$. If 3 is a cubic residue modulo $p$, then for any integer $k \geq 3$, we also have the third-order linear recurrence formula

$$
U_{-k}(p)=-\frac{3}{d} \cdot U_{-(k-1)}(p)+\frac{1}{\mathrm{~d} p} \cdot U_{-(k-3)}(p)
$$

where the first three terms are $U_{0}(p)=2 p^{3}-7 p^{2}$, $U_{-1}(p)=-p \cdot\left(2 p^{2}+p+d^{2}-2\right) / d$, and $U_{-2}(p)=1-3 p+$ $3 p\left(2 p^{2}+p-2\right) / d^{2}$.

Theorem 3. Let $p$ be an odd prime with $p \equiv 1 \bmod 3$. If 3 is not a cubic residue modulo $p$, then we have the identity

$$
\sum_{m=1}^{p-1}\left|\sum_{a=0}^{p-1} e\left(\frac{\mathrm{ma}^{3}}{p}\right)\right|^{2} \cdot\left|\sum_{a=0}^{p-1} e\left(\frac{\mathrm{ma}^{3}+a}{p}\right)\right|^{4}=p^{2}\left(4 p^{2}-19 p-d^{2}-1\right) .
$$

From our theorems, we may immediately deduce the following three corollaries:

Corollary 1. Let $p$ be an odd prime with $p \equiv 1 \bmod 3$, then we have

$$
\begin{aligned}
& \sum_{m=1}^{p-1}\left|\sum_{a=0}^{p-1} e\left(\frac{\mathrm{ma}^{3}}{p}\right)\right|^{2} \cdot\left|\sum_{a=0}^{p-1} e\left(\frac{\mathrm{ma}^{3}+a}{p}\right)\right|^{4} \\
& \quad= \begin{cases}p^{2}\left(4 p^{2}-22 p-d^{2}+2\right) & \text { if } 3 \text { is a cubic residue modulo } p, \\
p^{2}\left(4 p^{2}-19 p-d^{2}-1\right) & \text { if } 3 \text { is not a cubic residue modulo } p .\end{cases}
\end{aligned}
$$

Corollary 2. Let $p$ be a prime with $p \equiv 1 \bmod 3$. If 3 is a cubic residue modulo $p$, then we have

$$
\sum_{m=1}^{p-1}\left|\sum_{a=0}^{p-1} e\left(\frac{\mathrm{ma}^{3}}{p}\right)\right|^{4} \cdot\left|\sum_{a=0}^{p-1} e\left(\frac{\mathrm{ma}^{3}+a}{p}\right)\right|^{4}=p^{2}\left(12 p^{3}-66 p^{2}-9 \mathrm{~d}^{2} p+6 p+d^{2}\right)
$$


Corollary 3. Let $p$ be a prime with $p \equiv 1 \bmod 3$. If 3 is a cubic residue modulo $p$, then we have

$$
\sum_{m=1}^{p-1}\left|\frac{\sum_{a=0}^{p-1} e\left(\left(\mathrm{ma}^{3}+a\right) / p\right)}{\sum_{a=0}^{p-1} e\left(\mathrm{ma}^{3} / p\right)}\right|^{4}=\frac{27}{d^{4}} \cdot\left(2 p^{3}+p^{2}-2 p\right)-\frac{1}{d^{2}} \cdot\left(8 p^{2}+7 p-11\right)-1 .
$$

Some notes: first in Theorem 2 , if $(3, p-1)=1$, then the question we are discussing is trivial. Because in this case, we have

$$
\sum_{a=0}^{p-1} e\left(\frac{\mathrm{ma}^{3}}{p}\right)=\sum_{a=0}^{p-1} e\left(\frac{\mathrm{ma}}{p}\right)=0 .
$$

Second, if $p \equiv 1 \bmod 3$ and 3 is not a cubic residue modulo $p$, then we can only get the exact value of $U_{2}(p)$.

Third, the advantage of our work is that we completely solve the calculation problem of $U_{k}(p)$ with $p \equiv 1 \bmod 3$.

Fourth, the mean value estimation of the exponential sums is closely related to the upper and lower bounds of the individual exponential sums. So, by studying the mean value of the positive exponential sums, we can obtain a better upper bound estimation of the exponential sums. If we want to get its lower bound estimation of the exponential sums, we should study the negative power of the exponential sums. Our Theorems 1 and 2 address both types of problems.

Finally, for any fixed positive integer $h \geq 5$, whether there is a third-order linear recurrence formula for the hybrid power mean

$$
W_{k}(h, p)=\sum_{m=1}^{p-1}\left(\sum_{a=0}^{p-1} e\left(\frac{\mathrm{ma}^{3}}{p}\right)\right)^{k} \cdot\left(\sum_{b=0}^{p-1} e\left(\frac{\mathrm{mb}^{3}+b}{p}\right)\right)^{h}
$$

is an open problem, which is the limitation of our work. The other drawback, of course, is that we cannot compute all $U_{k}(p)$ when 3 is not a cubic residue modulo $p$. In fact, our ultimate goal is to obtain a precise calculation formula for
$W_{k}(h, p)$ for all positive integers $h \geq 5$. In the future, we will continue to improve the research in this aspect. It also requires us to continue to study.

\section{Several Lemmas}

To complete the proofs of our theorems, several simple lemmas are necessary. Hereafter, we will use many properties of the classical Gauss sums and the third-order character modulo $p$, all of which can be found in books concerning about Elementary Number Theory or Analytic Number Theory, such as references [14-16], so the related contents will not be repeated here. First we have the following:

Lemma 1. If $p$ is a prime with $p \equiv 1 \bmod 3$, then for any third-order character $\psi \bmod p$, we have the identity

$$
\sum_{m=1}^{p-1} \psi(m)\left(\sum_{a=0}^{p-1} e\left(\frac{m a^{3}+a}{p}\right)\right)^{3}=\bar{\psi}(3) \tau^{2}(\bar{\psi})-3 p \tau(\psi) .
$$

Proof. First applying trigonometric identity

$$
\sum_{m=1}^{q} e\left(\frac{\mathrm{nm}}{q}\right)= \begin{cases}q & \text { if } q \mid n, \\ 0 & \text { if } q \nmid n,\end{cases}
$$

and noting that $\psi^{3}=\chi_{0}$, the principal character modulo $p$, we have

$$
\begin{aligned}
\sum_{m=1}^{p-1} \psi(m)\left(\sum_{a=0}^{p-1} e\left(\frac{\mathrm{ma}^{3}+a}{p}\right)\right)^{3}= & \sum_{m=1}^{p-1} \psi(m)\left(\sum_{a=0}^{p-1} e\left(\frac{\mathrm{ma}^{3}+a}{p}\right)\right)^{2} \\
& +\sum_{m=1}^{p-1} \psi(m)\left(\sum_{a=0}^{p-1} e\left(\frac{\mathrm{ma}^{3}+a}{p}\right)\right)^{2}\left(\sum_{a=1}^{p-1} e\left(\frac{\mathrm{ma}^{3}+a}{p}\right)\right) \\
= & 2 \sum_{m=1}^{p-1} \psi(m) \sum_{a=1}^{p-1} e\left(\frac{\mathrm{ma}^{3}+a}{p}\right)+\sum_{m=1}^{p-1} \psi(m)\left(\sum_{a=1}^{p-1} e\left(\frac{\mathrm{ma}^{3}+a}{p}\right)\right)^{2} \\
& +\tau(\psi) \sum_{a=0}^{p-1} \sum_{b=0}^{p-1} \sum_{c=1}^{p-1} \bar{\psi}\left(a^{3}+b^{3}+c^{3}\right) e\left(\frac{a+b+c}{p}\right)
\end{aligned}
$$




$$
\begin{aligned}
= & -2 \tau(\psi)+\tau(\psi) \sum_{a=1}^{p-1} \bar{\psi}\left(a^{3}+1\right) \sum_{b=1}^{p-1} e\left(\frac{b(a+1)}{p}\right) \\
& +\tau(\psi) \sum_{a=0}^{p-1} \sum_{b=0}^{p-1} \bar{\psi}\left(a^{3}+b^{3}+1\right) \sum_{c=1}^{p-1} e\left(\frac{c(a+b+1)}{p}\right) \\
= & -2 \tau(\psi)-\tau(\psi) \sum_{a=1}^{p-1} \bar{\psi}\left(a^{3}+1\right)+p \tau(\psi) \sum_{a=0}^{p-1} \sum_{b=0}^{p-1} \bar{\psi}\left(a^{3}+b^{3}+1\right) \\
& -\tau(\psi) \sum_{a=0}^{p-1} \sum_{b=0}^{p-1} \bar{\psi}\left(a^{3}+b^{3}+1\right) \\
= & -2 \tau(\psi)-\tau(\psi) \sum_{a=1}^{p-1} \bar{\psi}\left(a^{3}+1\right)+p \tau(\psi) \sum_{a=0}^{p-1} \bar{\psi}\left(a^{3}-(a+1)^{3}+1\right) \\
& -\tau(\psi) \sum_{a=0}^{p-1} \sum_{b=0}^{p-1} \bar{\psi}\left(a^{3}+b^{3}+1\right) .
\end{aligned}
$$

Note that $\psi^{2}=\bar{\psi}$ and $\tau(\psi) \tau(\bar{\psi})=p$, and from the properties of Gauss sums and the characteristic function of the third-order character modulo $p$

$1+\psi(a)+\bar{\psi}(a)= \begin{cases}3, & \text { if } a \text { is a cubic residue modulo } p, \\ 0, & \text { if } a \text { is not a cubic residue modulo } p\end{cases}$

we have

$$
\begin{aligned}
\sum_{a=1}^{p-1} \bar{\psi}\left(a^{3}+1\right) & =\sum_{a=1}^{p-1} \bar{\psi}(a+1)(1+\psi(a)+\bar{\psi}(a)) \\
& =\sum_{a=1}^{p-1} \bar{\psi}(a+1)+\sum_{a=1}^{p-1} \bar{\psi}(1+\bar{a})+\sum_{a=1}^{p-1} \bar{\psi}\left(a^{2}+a\right) \\
& =-2+\frac{1}{\tau(\psi)} \sum_{b=1}^{p-1} \psi(b) \sum_{a=1}^{p-1} \bar{\psi}(a) e\left(\frac{b(a+1)}{p}\right) \\
& =-2+\frac{\tau^{2}(\bar{\psi})}{\tau(\psi)}=-2+\frac{\tau^{3}(\bar{\psi})}{p},
\end{aligned}
$$

$$
\begin{aligned}
\sum_{a=0}^{p-1} \bar{\psi}\left(a^{3}-(a+1)^{3}+1\right) & =\sum_{a=0}^{p-1} \bar{\psi}(-3 a(a+1)) \\
& =\bar{\psi}(3) \sum_{a=1}^{p-1} \bar{\psi}(a(a+1)) \\
& =\frac{\bar{\psi}(3) \tau^{3}(\bar{\psi})}{p}
\end{aligned}
$$

Since $\psi$ is a third-order character modulo $p$, for any integer $c$ with $(c, p)=1$, from the properties of the classical Gauss sums, we have

$$
\begin{aligned}
\sum_{a=0}^{p-1} e\left(\frac{\mathrm{ca}^{3}}{p}\right) & =1+\sum_{a=1}^{p-1}(1+\psi(a)+\bar{\psi}(a)) e\left(\frac{\mathrm{ca}}{p}\right) \\
& =\bar{\psi}(c) \tau(\psi)+\psi(c) \tau(\bar{\psi}) .
\end{aligned}
$$
get

From (22) and the properties of Gauss sums, then we can

$$
\begin{aligned}
\sum_{a=0}^{p-1} \sum_{b=0}^{p-1} \bar{\psi}\left(a^{3}+b^{3}+1\right)= & \frac{1}{\tau(\psi)} \sum_{c=1}^{p-1} \psi(c) \sum_{a=0}^{p-1} \sum_{b=0}^{p-1} e\left(\frac{\mathrm{ca}^{3}+\mathrm{cb}^{3}+c}{p}\right) \\
= & \frac{1}{\tau(\psi)} \sum_{c=1}^{p-1} \psi(c) e\left(\frac{c}{p}\right)\left(\sum_{a=0}^{p-1} e\left(\frac{\mathrm{ca}^{3}}{p}\right)\right)^{2} \\
= & \frac{1}{\tau(\psi)} \sum_{c=1}^{p-1} \psi(c) e\left(\frac{c}{p}\right)\left(\psi(c) \tau^{2}(\psi)\right. \\
& \left.+2 p+\bar{\psi}(c) \tau^{2}(\bar{\psi})\right) \\
= & \tau(\psi) \tau(\bar{\psi})+2 p-\frac{\tau^{3}(\bar{\psi})}{p}=3 p-\frac{\tau^{3}(\bar{\psi})}{p}
\end{aligned}
$$

Combining (18), (20), (21), and (23), we have the identity

$$
\sum_{m=1}^{p-1} \psi(m)\left(\sum_{a=0}^{p-1} e\left(\frac{m a^{3}+a}{p}\right)\right)^{3}=\bar{\psi}(3) \tau^{2}(\bar{\psi})-3 p \tau(\psi) .
$$

This proves Lemma 1.

Lemma 2. If $p$ is a prime with $p \equiv 1 \bmod 3$ and $\psi$ is any third-order character modulo $p$, then we have the identity

$$
\tau^{3}(\psi)+\tau^{3}(\bar{\psi})=\mathrm{d} p,
$$


where $\tau(\psi)$ denotes the classical Gauss sums, $d$ is uniquely determined by $4 p=d^{2}+27 b^{2}$, and $d \equiv 1 \bmod 3$.

Proof. See [3] or [11].

Lemma 3. If $p$ is a prime with $p \equiv 1 \bmod 3$, then for any third-order character $\psi \bmod p$, we have the identity

$$
\begin{aligned}
& \sum_{m=1}^{p-1} \psi(m)\left(\sum_{a=0}^{p-1} e\left(\frac{\mathrm{ma}^{3}+a}{p}\right)\right)^{4} \\
& \quad=(\bar{\psi}(3)-3 p-\bar{\psi}(3) p) \tau^{2}(\bar{\psi})-\mathrm{d} p \tau(\psi) .
\end{aligned}
$$

Proof. Note that the two-term exponential sums satisfies

$$
\sum_{a=0}^{p-1} e\left(\frac{\mathrm{ma}^{3}+a}{p}\right)=\sum_{a=0}^{p-1} e\left(\frac{-\mathrm{ma}^{3}-a}{p}\right) .
$$

So, from the properties of Gauss sums and Lemma 1, we have

$$
\begin{aligned}
\sum_{m=1}^{p-1} \psi(m)\left(\sum_{a=0}^{p-1} e\left(\frac{m a^{3}+a}{p}\right)\right)^{4} & \\
= & \sum_{m=1}^{p-1} \psi(m)\left(\sum_{a=0}^{p-1} e\left(\frac{\mathrm{ma}^{3}+a}{p}\right)\right)^{3}\left(\sum_{d=1}^{p-1} e\left(\frac{\mathrm{md}^{3}+d}{p}\right)\right) \\
& +\sum_{m=1}^{p-1} \psi(m)\left(\sum_{a=0}^{p-1} e\left(\frac{m a^{3}+a}{p}\right)\right)^{3} \\
= & \tau(\psi) \sum_{a=0}^{p-1} \sum_{b=0}^{p-1} \sum_{c=0}^{p-1} \bar{\psi}\left(a^{3}+b^{3}-c^{3}-1\right) \sum_{d=1}^{p-1} e\left(\frac{d(a+b-c-1)}{p}\right) \\
& +\bar{\psi}(3) \tau^{2}(\bar{\psi})-3 p \tau(\psi) \\
= & p \tau(\psi) \sum_{a=0}^{p-1} \sum_{b=0}^{p-1} \sum_{c=0}^{p-1} \bar{\psi}\left(a^{3}+b^{3}-c^{3}-1\right) \\
& +\bar{\psi}(3) \tau^{2}(\bar{\psi})-3 p \tau(\psi) \\
& -\tau(\psi) \sum_{a=0}^{p-1} \sum_{b=0}^{p-1} \sum_{c=0}^{p-1} \bar{\psi}\left(a^{3}+b^{3}-c^{3}-1\right) .
\end{aligned}
$$

From (22) and Lemma 2, we have

$$
\begin{aligned}
A^{3}(m) & =\left(\sum_{a=0}^{p-1} e\left(\frac{\mathrm{ma}^{3}}{p}\right)\right)^{3} \\
& =(\bar{\psi}(c) \tau(\psi)+\psi(c) \tau(\bar{\psi}))^{3} \\
& =\tau^{3}(\psi)+\tau^{3}(\bar{\psi})+3 \mathrm{pA}(m) \\
& =\mathrm{d} p+3 \mathrm{pA}(m) .
\end{aligned}
$$

It is clear that $A(m)$ is a real number, so from the properties of Gauss sums and (29), we have

$$
\begin{aligned}
& \tau(\psi) \sum_{a=0}^{p-1} \sum_{b=0}^{p-1} \sum_{c=0}^{p-1} \bar{\psi}\left(a^{3}+b^{3}-c^{3}-1\right) \\
& =\sum_{m=1}^{p-1} \psi(m) \sum_{a=0}^{p-1} \sum_{b=0}^{p-1} \sum_{c=0}^{p-1} e\left(\frac{m\left(a^{3}+b^{3}-c^{3}-1\right)}{p}\right) \\
& =\sum_{m=1}^{p-1} \psi(m) e\left(\frac{-m}{p}\right) A^{3}(m)=\sum_{m=1}^{p-1} \psi(m) e\left(\frac{-m}{p}\right)(\mathrm{d} p+3 p A(m)) \\
& =\mathrm{d} p \tau(\psi)+3 p \sum_{m=1}^{p-1} \psi(m)(\bar{\psi}(m) \tau(\psi)+\psi(m) \tau(\bar{\psi})) e\left(\frac{-m}{p}\right) \\
& =\mathrm{d} p \tau(\psi)-3 p \tau(\psi)+3 p \tau^{2}(\bar{\psi})=p(d-3) \tau(\psi)+3 p \tau^{2}(\bar{\psi}) .
\end{aligned}
$$

Note that the congruence $a+b \equiv c+1 \bmod p$ implies the congruence

$a^{3}+b^{3}-c^{3}-1 \equiv-3(a+b)(a-1)(b-1) \bmod p$.

So, we have

$$
\begin{aligned}
& \sum_{\substack{a=0 \\
a+b \equiv c+1 \bmod p \\
p-1}}^{p-1} \sum_{c=0}^{p-1} \bar{\psi}\left(a^{3}+b^{3}-c^{3}-1\right) \\
& =\sum_{a=0}^{p-1} \sum_{b=0}^{p-1} \bar{\psi}(-3(a+b)(a-1)(b-1)) \\
& =\bar{\psi}(3) \sum_{a=1}^{p-1} \sum_{b=1}^{p-1} \bar{\psi}((a+b+2) \mathrm{ab}) \\
& =\bar{\psi}(3) \sum_{a=1}^{p-1} \bar{\psi}(a) \sum_{b=1}^{p-1} \bar{\psi}(b(a+b+2)) .
\end{aligned}
$$

It is clear that

$$
\begin{aligned}
\sum_{b=1}^{p-1} \bar{\psi}(b(a+b+2)) & =\frac{1}{\tau(\psi)} \sum_{c=1}^{p-1} \psi(c) \sum_{b=1}^{p-1} \bar{\psi}(b) e\left(\frac{c(b+a+2)}{p}\right) \\
& =\frac{\tau(\bar{\psi})}{\tau(\psi)} \sum_{c=1}^{p-1} \psi^{2}(c) e\left(\frac{c(a+2)}{p}\right)=\frac{\tau^{2}(\bar{\psi})}{\tau(\psi)} \cdot \psi(a+2) .
\end{aligned}
$$


From (32) and (33), we have

$$
\begin{aligned}
\sum_{a=1}^{p-1} \sum_{b=1}^{p-1} \bar{\psi}((a+b+2) \mathrm{ab}) & =\frac{\tau^{2}(\bar{\psi})}{\tau(\psi)} \sum_{a=1}^{p-1} \bar{\psi}(a) \psi(a+2) \\
& =\frac{\tau^{2}(\bar{\psi})}{\tau(\psi)} \sum_{a=1}^{p-1} \psi(1+2 \bar{a}) \\
& =\frac{\tau^{2}(\bar{\psi})}{\tau(\psi)} \sum_{a=1}^{p-1} \psi(1+2 a)=-\frac{\tau^{2}(\bar{\psi})}{\tau(\psi)}
\end{aligned}
$$

Combining (28), (30), (32), and (34), we may immediately deduce that

$$
\sum_{m=1}^{p-1} \psi(m)\left(\sum_{a=0}^{p-1} e\left(\frac{\mathrm{ma}^{3}+a}{p}\right)\right)^{4}=(\bar{\psi}(3)-3 p-\bar{\psi}(3) p) \tau^{2}(\bar{\psi})-\mathrm{d} p \tau(\psi)
$$

This proves Lemma 3.

Lemma 4. If $p$ is a prime with $p \equiv 1 \bmod 3$, then we have the identity

$$
\sum_{m=1}^{p-1}\left|\sum_{a=0}^{p-1} e\left(\frac{m a^{3}+a}{p}\right)\right|^{4}=2 p^{3}-7 p^{2}
$$

Proof. See Zhang and Zhang [13].

\section{Proofs of the Theorems}

Now, we prove our main results. First, we prove Theorem 1. If 3 is a cubic residue $\bmod p$, then $\psi(3)=1$. From $(22)$, Lemmas 2 and 3, we have

$$
\begin{aligned}
U_{1}(p) & =\sum_{m=1}^{p-1} A(m)\left(\sum_{a=0}^{p-1} e\left(\frac{\mathrm{ma}^{3}+a}{p}\right)\right)^{4} \\
& =\sum_{m=1}^{p-1}(\bar{\psi}(m) \tau(\psi)+\psi(m) \tau(\bar{\psi}))\left(\sum_{a=0}^{p-1} e\left(\frac{\mathrm{ma}^{3}+a}{p}\right)\right)^{4} \\
& =\tau(\bar{\psi})\left((1-4 p) \tau^{2}(\bar{\psi})-\mathrm{d} p \tau(\psi)\right)+\tau(\psi)\left((1-4 p) \tau^{2}(\psi)-\mathrm{d} p \tau(\bar{\psi})\right) \\
& =(1-4 p)\left(\tau^{3}(\psi)+\tau^{3}(\bar{\psi})\right)-2 \mathrm{~d} p^{2}=-\mathrm{d} p(6 p-1) .
\end{aligned}
$$

Applying (22), Lemmas 2-4, we have

$$
\begin{aligned}
U_{2}(p)= & \sum_{m=1}^{p-1}\left(\sum_{a=0}^{p-1} e\left(\frac{\mathrm{ma}^{3}}{p}\right)\right)^{2}\left(\sum_{a=0}^{p-1} e\left(\frac{\mathrm{ma}^{3}+a}{p}\right)\right)^{4} \\
= & \sum_{m=1}^{p-1}\left(\psi(m) \tau^{2}(\psi)+2 p+\bar{\psi}(m) \tau^{2}(\bar{\psi})\right)\left(\sum_{a=0}^{p-1} e\left(\frac{\mathrm{ma}^{3}+a}{p}\right)\right)^{4} \\
= & 2 p\left(2 p^{3}-7 p^{2}\right)+\tau^{2}(\psi)\left((1-4 p) \tau^{2}(\bar{\psi})-\mathrm{d} p \tau(\psi)\right) \\
& +\tau^{2}(\bar{\psi})\left((1-4 p) \tau^{2}(\psi)-\mathrm{d} p \tau(\bar{\psi})\right) \\
= & p^{2}\left(4 p^{2}-22 p-d^{2}+2\right) .
\end{aligned}
$$


Applying (29), (37), Lemmas 3 and 4, we also have

$$
\begin{aligned}
U_{3}(p) & =\sum_{m=1}^{p-1}\left(\sum_{a=0}^{p-1} e\left(\frac{\mathrm{ma}^{3}}{p}\right)\right)^{3}\left(\sum_{a=0}^{p-1} e\left(\frac{\mathrm{ma}^{3}+a}{p}\right)\right)^{4} \\
& =\sum_{m=1}^{p-1}(\mathrm{~d} p+3 \mathrm{pA}(m))\left(\sum_{a=0}^{p-1} e\left(\frac{\mathrm{ma}^{3}+a}{p}\right)\right)^{4} \\
& =\mathrm{d} p\left(2 p^{3}-7 p^{2}\right)-3 \mathrm{~d} p^{2}(6 p-1) \\
& =\mathrm{d} p^{2}\left(2 p^{2}-25 p+3\right) .
\end{aligned}
$$

If $k \geq 3$, then from (29) and the definition of $U_{k}(p)$, we have

$$
\begin{aligned}
U_{k}(p) & =\sum_{m=1}^{p-1} A^{k}(m)\left(\sum_{a=0}^{p-1} e\left(\frac{\mathrm{ma}^{3}+a}{p}\right)\right)^{4} \\
& =\sum_{m=1}^{p-1} A^{k-3}(m)(\mathrm{d} p+3 \mathrm{pA}(m))\left(\sum_{a=0}^{p-1} e\left(\frac{\mathrm{ma}^{3}+a}{p}\right)\right)^{4} \\
& =3 \mathrm{pU}_{k-2}(p)+\mathrm{dpU}_{k-3}(p) .
\end{aligned}
$$

Combining Lemma 4, (37)-(40), we complete the proof of Theorem 1.
Note that identity $A^{3}(m)=\mathrm{d} p+3 \mathrm{pA}(m)$, so we have $A^{-1}(m)=\left(A^{2}(m)-3 p\right) / \mathrm{d} p, \quad A^{-2}(m)=\left(A(m)-3 \mathrm{pA}^{-1}\right.$ $(m)) / \mathrm{d} p$, and $A^{-3}(m)=(1 / \mathrm{d} p)-(3 / d) \cdot A^{-2}(m)$.

From Lemma 4 and Theorem 1, we have the third-order linear recurrence formula:

$$
\begin{aligned}
U_{0}(p) & =2 p^{3}-7 p^{2} \\
U_{-1}(p) & =\frac{1}{d p}\left(U_{2}(p)-3 p U_{0}(p)\right)=\frac{-p \cdot\left(2 p^{2}+p+d^{2}-2\right)}{d} \\
U_{-2}(p) & =\frac{1}{d p}\left(U_{1}(p)-3 p U_{-1}(p)\right) \\
& =\frac{(1-3 p) d^{2}+3 p\left(2 p^{2}+p-2\right)}{d^{2}}
\end{aligned}
$$

If $k \geq 3$, then we have the third-order linear recurrence formula

$$
U_{-k}(p)=-\frac{3}{d} \cdot U_{-(k-1)}(p)+\frac{1}{\mathrm{~d} p} \cdot U_{-(k-3)}(p) .
$$

This proves Theorem 2 .

Now we prove Theorem 3. If $p \equiv 1 \bmod 3$ and 3 is not a cubic residue modulo $p$, then we have $\psi(3)+\bar{\psi}(3)+1=0$ or $\psi(3)+\bar{\psi}(3)=-1$. From (22), Lemmas $2-4$, we have

$$
\begin{aligned}
\sum_{m=1}^{p-1}\left|\sum_{a=0}^{p-1} e\left(\frac{\mathrm{ma}^{3}}{p}\right)\right|^{2} \cdot\left|\sum_{a=0}^{p-1} e\left(\frac{\mathrm{ma}^{3}+a}{p}\right)\right|^{4} \\
=\sum_{m=1}^{p-1}\left(\psi(m) \tau^{2}(\psi)+2 p+\bar{\psi}(m) \tau^{2}(\bar{\psi})\right)\left(\sum_{a=0}^{p-1} e\left(\frac{\mathrm{ma}^{3}+a}{p}\right)\right)^{4} \\
=\tau^{2}(\psi)\left((\bar{\psi}(3)-3 p-\bar{\psi}(3) p) \tau^{2}(\bar{\psi})-\mathrm{d} p \tau(\psi)\right)+2 p\left(2 p^{3}-7 p^{2}\right) \\
\quad+\tau^{2}(\bar{\psi})\left((\psi(3)-3 p-\psi(3) p) \tau^{2}(\psi)-\mathrm{d} p \tau(\bar{\psi})\right) \\
=(\psi(3)+\bar{\psi}(3)-6 p-(\psi(3)+\bar{\psi}(3)) p) p^{2}-\mathrm{d} p\left(\tau^{3}(\psi)+\tau^{3}(\bar{\psi})\right)+2 p^{3}(2 p-7) \\
=-(5 p+1) p^{2}-d^{2} p^{2}+2 p^{3}(2 p-7)=p^{2}\left(4 p^{2}-19 p-d^{2}-1\right) .
\end{aligned}
$$

This completes the proofs of all our results.

\section{Conclusion}

This paper mainly proposed three theorems, which are all closely related to the hybrid power mean of the two-term exponential sums and the classical cubic Gauss sums. Theorems 1 and 2 obtained two interesting third-order linear recurrence formulas of $U_{k}(p)$ for $k \geq 0$ and $k \leq 0$, respectively, providing 3 is a cubic residue modulo $p$.
However, when 3 is not a cubic residue modulo $p$, we gave an exact calculating formula for $U_{2}(p)$ in Theorem 3. In general, this work not only generalized the results in reference [12] but also provided some new ideas and methods for the further study of such problems.

\section{Data Availability}

No data were used to support this study. 


\section{Conflicts of Interest}

The authors declare that there are no conflicts of interest regarding the publication of this paper.

\section{Authors' Contributions}

All authors have equally contributed to this work. All authors read and approved the final manuscript.

\section{Acknowledgments}

This work was supported by the Y. S. T. N. S. P (2019KJXX076), the N. S. B. R. P. (2019JM-207) of Shaanxi Province, and the N. S. F. (11771351) of P. R. China.

\section{References}

[1] L. Chen and J. Y. Hu, "A linear recurrence formula involving cubic Gauss sums and Kloosterman sums," Acta Mathematica Sinica, vol. 61, pp. 67-72, 2018.

[2] X. X. Li and J. Y. Hu, "The hybrid power mean quartic Gauss sums and Kloosterman sums," Open Mathematics, vol. 15, pp. 151-156, 2017.

[3] W. P. Zhang and J. Y. Hu, "The number of solutions of the diagonal cubic congruence equation $\bmod p$," Mathematical Reports, vol. 20, pp. 73-80, 2018.

[4] Z. Y. Chen and W. P. Zhang, "On the fourth-order linear recurrence formula related to classical Gauss sums," Open Mathematics, vol. 15, pp. 1251-1255, 2017.

[5] S. M. Shen and W. P. Zhang, "On the quartic Gauss sums and their recurrence property," Advances in Difference Equations, vol. 2017, p. 43, 2017.

[6] X. Y. Liu and W. P. Zhang, "On the high-power mean of the generalized Gauss sums and Kloosterman sums," Mathematics, vol. 7, no. 10, p. 907, 2019.

[7] W. P. Zhang and H. N. Liu, "On the general Gauss sums and their fourth power mean," Osaka Journal of Mathematics, vol. 42, pp. 189-199, 2005.

[8] D. Han, "A Hybrid mean value involving two-term exponential sums and polynomial character sums," Czechoslovak Mathematical Journal, vol. 64, pp. 53-62, 2014.

[9] W. P. Zhang and D. Han, "On the sixth power mean of the two-term exponential sums," Journal of Number Theory, vol. 136, pp. 403-413, 2014.

[10] B. C. Berndt and R. J. Evans, "The determination of Gauss sums," Bulletin of the American Mathematical Society, vol. 5, no. 2, pp. 107-128, 1981.

[11] B. C. Berndt and R. J. Evans, "Sums of Gauss, Jacobi, and Jacobsthal," Journal of Number Theory, vol. 11, pp. 349-389, 1979.

[12] L. Chen and Z. Y. Chen, "Some new hybrid power mean formulae of trigonometric sums," Advances in Differences Equation, vol. 2020, p. 220, 2020.

[13] H. Zhang and W. P. Zhang, "The fourth power mean of twoterm exponential sums and its application," Mathematical Reports, vol. 19, pp. 75-83, 2017.

[14] T. M. Apostol, Introduction to Analytic Number Theory, Springer-Verlag, New York, NY, USA, 1976.

[15] W. P. Zhang and H. L. Li, Elementary Number Theory, Shaanxi Normal University Press, Xi'an, China, 2013.

[16] L. K. Hua, Introduction to Number Theory, Science Press, Beijing, China, 1979. 